\title{
On the spin evolution of neutron stars in pre-low-mass X-ray binaries
}

\author{
S. B. Popov ${ }^{1,2, \star}$ \\ ${ }^{1}$ Università di Padova, Dipartimento di Fisica, via Marzolo 8, 35131 Padova, Italy \\ 2 Sternberg Astronomical Institute, Universitetski pr. 13, 119992 Moscow, Russia \\ Received 17 November 2003 / Accepted 2 February 2004

\begin{abstract}
We present a simple model for the evolutionary states of neutron stars in pre-low-mass X-ray binaries during the main sequence stage of donor stars. It is shown that for typical parameters some of the neutron stars in these systems cannot $10^{13} \mathrm{G}$ and higher and/or in close systems with orbital periods of about a few days and shorter.
\end{abstract} \\ accrete matter from winds of their companions in a stable way. Accretors are found if neutron stars have magnetic fields about
}

Key words. stars: binaries (including multiple): close - stars: neutron - stars: evolution - X-rays: stars

\section{Introduction}

The increased ability to observe very faint $\mathrm{X}$-ray sources with modern X-ray observatories (XMM-Newton, Chandra) prompted new interest in systems with low accretion rates $\sim 10^{10}-10^{12} \mathrm{~g} \mathrm{~s}^{-1}$ like isolated neutron stars (NSs) or NSs in detached binaries. Among isolated NSs the fraction of accreting objects is not expected to be high, and accretors are expected to be dim (see Popov et al. 2003 for a review and discussion).

Recently Willems \& Kolb (2003) discussed the evolution and observational appearance of pre-low-mass X-ray binaries (pre-LMXBs) (see also Belczynski \& Taam 2003). Pre-LMXBs by definition represent an evolutionary state prior to LMXB. These systems are detached binaries in which NSs accrete matter from the stellar wind of its companion and have detectable accretion luminosity.

Willems and Kolb found two maxima in the distribution of accretion luminosity of NSs in pre-LMXBs at $\sim 10^{31}$ and $\sim 10^{28} \mathrm{erg} \mathrm{s}^{-1}$. The second maximum corresponds to very small accretion rates similar to those of isolated NSs accreting the interstellar medium. One can suspect that for such small mass flow a NS can avoid accretion completely. Willems \& Kolb (2003) did not explore the evolutionary state of NSs in pre-LMXBs, assuming that all of them can accrete. Here in a very simple model we try to calculate whether NSs in pre-LMXBs can reach the stage of accretion or if they are at the propeller or ejector stages (see Lipunov 1992 for a detailed description of the different stages and Popov et al. 2003 for a short introduction).

\footnotetext{
* e-mail: polar@sai.msu.ru
}

\section{The model}

We assume that the evolution of a NS starts at the stage of ejection (i.e. that the initial period of the NS is short enough), then continues to the propeller stage (including subsonic propeller, see Davies \& Pringle 1981) and finally reaches the stage of accretion. Stages are separated by critical periods (see the detailed derivation in Lipunov 1992). A NS is born as an ejector and starts to slow down. The ejector stage ends when $P=P_{\mathrm{E}}$ :

$P_{\mathrm{E}}=2 \pi\left(\frac{2 \mu^{2}}{c^{4} V \dot{M}_{\mathrm{acc}}}\right)^{1 / 4} \approx 10 \mu_{30}^{1 / 2} n^{-1 / 4} V_{6}^{1 / 2} \mathrm{~s}, R_{\mathrm{l}}<R_{\mathrm{G}}$

Here $R_{1}=c P / 2 \pi=4.8 \times 10^{9} \mathrm{Pcm}-$ light cylinder radius, $R_{\mathrm{G}}=2 G M_{\mathrm{NS}} / V^{2}=3.7 \times 10^{14} V_{6}^{-2} \mathrm{~cm}-$ radius of accretion, $\mu_{30}=\mu / 10^{30} \mathrm{G} \mathrm{cm}^{3}$ - magnetic moment of NS, $V_{6}=V_{\text {Total }} / 10^{6} \mathrm{~cm} \mathrm{~s}^{-1}, V_{\text {Total }}=\sqrt{V_{\text {wind }}^{2}+\left(V_{\text {orb }}\right)^{2}}, n-$ number density of the stellar wind at the NS position. In all formulae here and below we assume the NS' moment of inertia to be $10^{45} \mathrm{~g} \mathrm{~cm}^{2}$.

Note that the condition $R_{\mathrm{l}}<R_{\mathrm{G}}$ is not necessary fulfilled in the binaries we are going to study. We discuss this topic in Sect. 4.

Subsonic and supersonic propellers are separated by the condition of equality of the co-rotation radius, $R_{\mathrm{co}}=$ $\left(G M P^{2} / 4 \pi^{2}\right)^{1 / 3}$ and radius of the magnetosphere, $R_{\mathrm{A}}$. It corresponds to a critical period:

$$
\begin{aligned}
P_{\mathrm{ss}} & =2^{5 / 14} \pi\left(G M_{\mathrm{NS}}\right)^{-5 / 7}\left(\mu^{2} / \dot{M}_{\mathrm{acc}}\right)^{3 / 7} \\
& \approx 300 \mu_{30}^{6 / 7} n^{-3 / 7} V_{6}^{9 / 7} \mathrm{~s}, R_{\mathrm{A}}<R_{\mathrm{G}}
\end{aligned}
$$

For some systems the relation $R_{\mathrm{A}}<R_{\mathrm{G}}$ can be violated. We discuss this in Sect. 4. 
Accretion starts when $P=P_{\text {br }}$ (unless a NS is at the georotator stage).

$P_{\text {br }}=6 \times 10^{6} \mu_{30}^{16 / 21} \dot{M}_{8}^{-5 / 7} m^{-4 / 21} \mathrm{~s}$.

Here $\dot{M}_{8}=\dot{M}_{\text {acc }} / 10^{8} \mathrm{~g} \mathrm{~s}^{-1}-$ accretion rate, $m=M_{\mathrm{NS}} / M_{\odot}$. We use the numerical coefficient $6 \times 10^{6}$ in Eq. (1) following Davies \& Pringle (but scaling it to the value of $\dot{M}_{\text {acc }}$ typical for pre-LMXBs). Ikhsanov (2003) suggested that it should be a factor 7.5 larger. If it is correct, then the time scale of the subsonic propeller stage is longer. However, this stage is not well understood, and we try to be conservative and whenever possible choose values that lower the timescales of pre-accretion stages.

The output of our calculations consists of two timescales: the ejector time scale, $t_{\mathrm{E}}$, and the propeller timescale, $t_{\mathrm{P}}$ (see Prokhorov et al. 2002; Lipunov 1992 for more details).

At the ejector stage a NS is spinning down according to the magneto-dipole formula (approximatelly $P=3 \times 10^{-4} \mu_{30} t_{\mathrm{yr}}^{1 / 2}$ ). The propeller stage consists of two distinct phases: the supersonic propeller and the subsonic propeller (see Davies \& Pringle 1981; Ikhsanov 2003), so we define $t_{\mathrm{P}}=t_{\text {super }}+t_{\text {sub }}$. Actually, there also should be an intermediate stage between the supersonic and subsonic propeller, when turbulence becomes supersonic above the magnetospheric radius, $R_{\mathrm{A}}$, but inside $R_{\mathrm{G}}$. Spin-down during this intermediate phase should be similar to the subsonic propeller rate, so they are united here. The supersonic propeller is a relatively short stage (normally shorter than each of the ejector and propeller stages). For the supersonic propeller we choose a very efficient spin-down (see Popov et al. 2000).

We can write the following equations for the duration of the ejector stage $\left(t_{\mathrm{E}}\right)$ and supersonic $\left(t_{\text {super }}\right)$ and subsonic $\left(t_{\text {sub }}\right)$ propeller stages:

$t_{\mathrm{E}}=0.8 \times 10^{9} \mu_{30}^{-1} n^{-1 / 2} V_{6} \mathrm{yr}$,

$t_{\text {super }}=1.3 \times 10^{6} \mu_{30}^{-8 / 7} n^{-3 / 7} V_{6}^{9 / 7} \mathrm{yr}$,

$t_{\mathrm{sub}}=10^{3} \times \mu_{30}^{-2} m P_{\mathrm{br}} \mathrm{yr}$.

Equation (2) is taken following Popov et al. (2000), Eq. (3) from Shakura (1975), and Eq. (4) from Davies \& Pringle (1981).

To calculate the orbital velocity, $V_{\text {orb}}$, we assume circular orbits and equal masses $M_{\mathrm{NS}}=M_{*}=1.4 M_{\odot}$ (Willems \& Kolb 2003 discuss $M_{\odot}<M_{*}<2 M_{\odot}$ ). In our calculations the orbital velocity is always smaller than the stellar wind velocity.

To calculate the accretion rate we use the following equation (see Willems \& Kolb 2003):

$\dot{M}_{\mathrm{acc}}=\frac{3}{16}\left(\frac{R_{*}}{a}\right)^{2} \frac{q^{2}}{\beta^{4}}\left(1+\frac{1+q}{2 \beta^{2}} \frac{R_{*}}{a}\right)^{-3 / 2} \dot{M}_{*}$.

Here $R_{*}$ - stellar radius, $q=M_{\mathrm{NS}} / M_{*}, \dot{M}_{*}-$ the rate of stellar wind mass loss. We used several different values for $\dot{M}_{*}$ and $V_{\text {wind }}=\beta \sqrt{2 G M_{*} / R_{*}}$ (see tables, $330 \mathrm{~km} \mathrm{~s}^{-1}$ corresponds to $\beta=0.5,660 \mathrm{~km} \mathrm{~s}^{-1}-$ to $\beta=1$ and $1320 \mathrm{~km} \mathrm{~s}^{-1}$ to $\beta=2$ ). Note that actual accretion rate can be lower than this value due

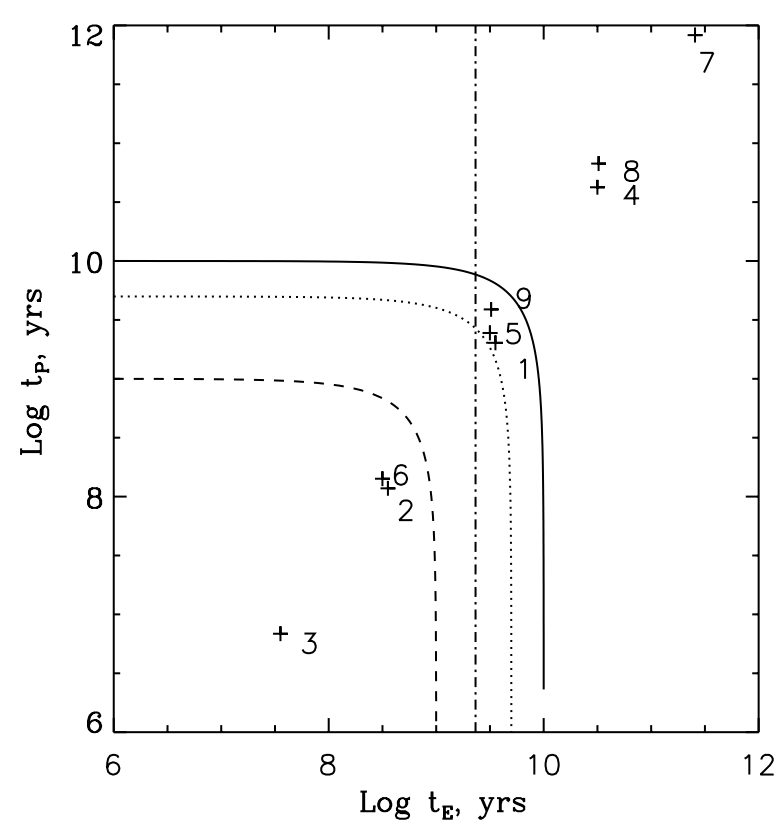

Fig. 1. Crosses with numbers represent different set of parameters of NSs (see Table 1). The three curves correspond to $t_{\text {Total }}=t_{\mathrm{E}}+t_{\mathrm{P}}$ equal to $1 \mathrm{Gyr}$ (dashed), $5 \mathrm{Gyr}$ (dotted) and $10 \mathrm{Gyr}$ (solid).The vertical line corresponds to $t_{\mathrm{E}}=2.3 \times 10^{9} \mathrm{yr}$.

to heating, magnetospheric and hydrodynamical effects (see discussion in Perna et al. 2003; Popov et al. 2003).

To derive many of the equations in this paper we will use the following relation which parametrises the accretion rate by $n$ and $V_{\text {Total }}$ :

$\dot{M}_{\mathrm{acc}}=7 \times 10^{11} n V_{6}^{-3} \mathrm{~g} \mathrm{~s}^{-1}$.

$n=\rho m_{\mathrm{p}}^{-1}, \rho=\dot{M}_{*} /\left(4 \pi a^{2} V_{\text {wind }}\right)-$ density of matter, $m_{\mathrm{p}}-$ proton mass.

For $\dot{M}_{*}$ on the main sequence stage Willems \& Kolb (2003) used the value $10^{-13} M_{\odot} \mathrm{yr}^{-1}$. We follow that choice and also check the higher value $10^{-12} M_{\odot} \mathrm{yr}^{-1}$, which was also discussed by them. Definitely for smaller $\dot{M}_{*}$ all timescales $\left(t_{\mathrm{E}}\right.$, $t_{\text {super }}, t_{\text {sub }}$ ) become longer; for higher $\dot{M}_{*}$ they become shorter.

Mass loss can be increased significantly when a donor star leaves the main sequence, but we do not follow these stages in our analysis. Also we do not discuss the possibility of formation of a binary system due to capturing of a companion (in that case the picture could be completely different as far as an old evolved NS can be captured).

Our model is a very simple one but our aim is just to illustrate the importance of the effects of magnetorotational evolution of NSs in pre-LMXBs. Discussion on possible complications can be found in Sect. 4 .

\section{Results}

Our main results are presented in the Figs. 1 and 2. Each point represents a model with particular set of parameters (see tables). The three lines correspond to the total time of evolution prior to the stage of accretion $\left(t_{\text {Total }}=t_{\mathrm{E}}+t_{\mathrm{P}}\right)$ equal to 1,5 and 10 billion years. 


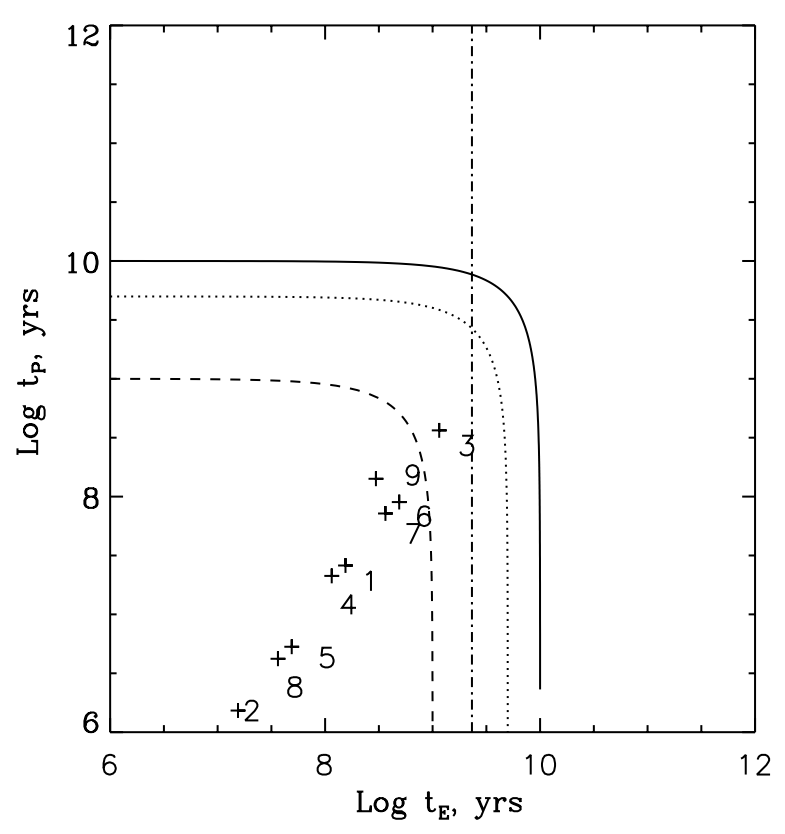

Fig. 2. Same as in Fig. 1 for parameters in Table 2.

Note that $t_{\mathrm{P}} \propto t_{\mathrm{E}}^{a}, a \approx 1.3$. This happens because in Eq. (2) $t_{\mathrm{E}} \propto \mu^{-1} n^{-1 / 2}$ and $V_{\text {Total }}$ is not varied significantly in our modeling when $V_{\text {wind }}>V_{\text {orb }}$. If we substitute Eq. (1) into Eq. (4), then we have $t_{\text {sub }} \propto \mu^{-26 / 21} n^{-5 / 7}$. (Remember that in $t_{\mathrm{P}}$ we have $t_{\text {super }} \ll t_{\text {sub }}$ [see tables].) As we see we have nearly $t_{\mathrm{P}} \propto t_{\mathrm{E}}^{1.3}$.

The vertical line in the figures corresponds to $t_{\mathrm{E}}=2.3 \times$ $10^{9} \mathrm{yr}$ - the main sequence lifetime of a star with $M_{*}=$ $1.4 M_{\odot}$. (The main sequence lifetime roughly can be estimated as: $\log t_{\mathrm{MS}}=9.9-3.8 \log \left(M_{*} / M_{\odot}\right)+\log ^{2}\left(M_{*} / M_{\odot}\right)$, see for example Lipunov 1992.) If for some reason the propeller stage appears to be very short (for example, the subsonic propeller is not operating) then NSs to the left of this line can accrete.

As one can see most of the models from Table 1 have $t_{\text {Total }}>2.3 \times 10^{9} \mathrm{yr}$. It means that these NSs cannot accrete. Accretion is more probable onto strongly magnetized NSs as their spin-down timescales are shorter at both the ejector and propeller stages. To allow accretion with a rate $\sim 10^{10} \mathrm{~g} \mathrm{~s}^{-1}$, a NS with $B \sim 10^{12} \mathrm{G}$ should be slowed down to very long periods $\sim 10^{5} \mathrm{~s}$, which takes a long time.

In the Fig. 2 we present models with $t_{\text {Total }}<5 \times 10^{9}$ yr. These are systems with strongly magnetized NSs or/and with shorter orbital periods or/and with lower wind velocity.

\section{Discussion and conclusions}

Our calculations were made for constant magnetic fields. As was shown by Colpi et al. (1998), Livio et al. (1998), and Popov \& Prokhorov (2000), realistic parameters of decay in the case of isolated NSs normally make the propeller stage longer. Parameters of the magneto-rotational evolution of isolated NSs and NSs in pre-LMXBs are very similar (for example, there is no huge accretion to speed-up the process of field decay), so the results for the former ones can be applied to the
Table 1. Parameters for the models in Fig. 1.

\begin{tabular}{|c|c|c|c|c|c|c|}
\hline $\begin{array}{c}\dot{M}_{*}, \\
M_{\odot} \mathrm{yr}^{-1} \\
\end{array}$ & $\begin{array}{l}\dot{M}_{\mathrm{acc}}, \\
\mathrm{g} \mathrm{s}^{-1}\end{array}$ & $\mu_{30}$ & $\begin{array}{l}T_{\text {orb }}, \\
\text { days }\end{array}$ & $\begin{array}{c}V_{\text {wind }}, \\
\mathrm{km} \mathrm{s}^{-1}\end{array}$ & $\frac{t_{\text {super }}}{t_{\text {sub }}}$ & $\begin{array}{l}\text { Mod. } \\
\text { num. }\end{array}$ \\
\hline $10^{-13}$ & $3.8 \times 10^{10}$ & 0.1 & 1 & 660 & 0.03 & 1 \\
\hline $10^{-13}$ & $3.8 \times 10^{10}$ & 1 & 1 & 660 & 0.04 & 2 \\
\hline $10^{-13}$ & $3.8 \times 10^{10}$ & 10 & 1 & 660 & 0.04 & 3 \\
\hline $10^{-13}$ & $5.2 \times 10^{8}$ & 0.1 & 30 & 660 & 0.008 & 4 \\
\hline $10^{-13}$ & $5.2 \times 10^{8}$ & 1 & 30 & 660 & 0.01 & 5 \\
\hline $10^{-13}$ & $5.2 \times 10^{8}$ & 10 & 30 & 660 & 0.01 & 6 \\
\hline $10^{-13}$ & $5.0 \times 10^{6}$ & 0.1 & 700 & 660 & 0.003 & 7 \\
\hline $10^{-13}$ & $5.0 \times 10^{6}$ & 1 & 1000 & 660 & 0.003 & 8 \\
\hline $10^{-13}$ & $5.0 \times 10^{6}$ & 10 & 1000 & 660 & 0.003 & 9 \\
\hline
\end{tabular}

Table 2. Parameters for the models in Fig. 2.

\begin{tabular}{|c|c|c|c|c|c|c|}
\hline $\begin{array}{c}\dot{M}_{*}, \\
M_{\odot} \mathrm{yr}^{-1}\end{array}$ & $\begin{array}{l}\dot{M}_{\text {acc }}, \\
\mathrm{g} \mathrm{s}^{-1}\end{array}$ & $\mu_{30}$ & $\begin{array}{l}T_{\text {orb }}, \\
\text { days }\end{array}$ & $\begin{array}{c}V_{\text {wind }}, \\
\mathrm{km} \mathrm{s}^{-1}\end{array}$ & $\frac{t_{\text {super }}}{t_{\text {sub }}}$ & $\begin{array}{l}\text { Mod. } \\
\text { num. }\end{array}$ \\
\hline $10^{-13}$ & $3.3 \times 10^{11}$ & 1 & 1 & 330 & 0.07 & 1 \\
\hline $10^{-13}$ & $3.3 \times 10^{11}$ & 10 & 1 & 330 & 0.08 & 2 \\
\hline $10^{-13}$ & $7.6 \times 10^{9}$ & 1 & 30 & 330 & 0.02 & 3 \\
\hline $10^{-13}$ & $7.6 \times 10^{9}$ & 10 & 30 & 330 & 0.03 & 4 \\
\hline $10^{-12}$ & $3.3 \times 10^{12}$ & 1 & 1 & 330 & 0.13 & 5 \\
\hline $10^{-12}$ & $3.3 \times 10^{12}$ & 0.1 & 1 & 330 & 0.1 & 6 \\
\hline $10^{-12}$ & $7.6 \times 10^{10}$ & 1 & 30 & 330 & 0.04 & 7 \\
\hline $10^{-12}$ & $7.6 \times 10^{10}$ & 10 & 30 & 330 & 0.05 & 8 \\
\hline $10^{-12}$ & $2.9 \times 10^{10}$ & 1 & 1 & 1320 & 0.03 & 9 \\
\hline
\end{tabular}

latter (and vice versa) ${ }^{1}$. In that sense our estimates of $t_{\mathrm{E}}$ and $t_{\mathrm{P}}$ for $B=$ const should be lower limits if the field is decaying. For decayed fields most NSs in pre-LMXBs can be expected to stay on the propeller stage. For high $V_{\text {wind }}$ a NS instead of accretion can stay on the so-called georotator stage (Lipunov 1992). This happens for $V_{\text {Total }} \gtrsim 470 \mu_{30}^{-1 / 5} n^{1 / 10} \mathrm{~km} \mathrm{~s}^{-1}$ (see Popov et al. 2003).

In this short illustrative note we neglect several effects that can change characteristic periods and radii or/and spin-down rates. Most of the time scales used above can be considered only as rough estimates (see discussion in Popov et al. 2003). However, we believe that qualitatively our results are valid, as our main aim is only to illustrate the importance of the effects of magneto-rotational evolution of NSs in pre-LMXBs.

Let us briefly discuss possible violations of the relations $R_{1}<R_{\mathrm{G}}, R_{\mathrm{A}}<R_{\mathrm{G}}$ (for details see Lipunov 1992). We start with the relation between $R_{1}$ and $R_{\mathrm{G}}$. For high magnetic fields $P_{\mathrm{E}}$ becomes large. It necessarily means large $R_{\mathrm{l}}$. On the other hand $V_{\text {wind }}$ is also large, which means small $R_{\mathrm{G}}$. For systems 3 , 6, 8, 9 in Table 1 and for system 9 in Table $2, R_{1}>R_{\mathrm{G}}$ at

1 We assume that isolated NSs and NSs in binaries are born rapidly rotating. However, this is a matter for discussion. 
the moment of transition from ejector to propeller. In that case equations for $P_{\mathrm{E}}$ and $t_{\mathrm{E}}$ are the following:

$P_{\mathrm{E} 2} \approx 180 \mu_{30}^{1 / 3} n^{-1 / 3} V_{6}^{-1 / 3} \mathrm{~s}$,

$t_{\mathrm{E} 2} \approx 3.5 \times 10^{11} \mu_{30}^{-4 / 3} n^{-1 / 3} V_{6}^{-2 / 3} \mathrm{yr}$.

For large velocities and magnetic fields, normally $t_{\mathrm{E}}>t_{\mathrm{E} 2}$, so the stage of ejection is shorter for $R_{\mathrm{l}}>R_{\mathrm{G}}$ (compare Eqs. (2), (6)). For our investigation it is crucial to know if this effect is strong enough to let the system cross the line $t_{\mathrm{E}}=$ $2.3 \times 10^{9} \mathrm{yr}$ on the figures. Only for model 9 from the Table 1 is this the case (in the figures all data points are plotted for $R_{1}<$ $R_{\mathrm{G}}$ ). For all others this shortening of $t_{\mathrm{E}}$ is not strong enough to change the conclusions made here. Also we want to mention that if a system leaves the ejector stage after the violation $R_{1}<R_{\mathrm{G}}$ then the accretion rate is expected to be very low.

Now let us discuss the relation between $R_{\mathrm{A}}$ and $R_{\mathrm{G}}$. Often $R_{\mathrm{A}}<R_{\mathrm{G}}$ (for example it is so for all observed bright $\mathrm{X}$-ray binaries, for isolated NSs which can become accretors etc.). For systems with highly magnetized NSs and high wind velocities it is possible that $R_{\mathrm{A}}>R_{\mathrm{G}}$. It is important for example to estimate the critical period $P_{\mathrm{ss}}$ and $t_{\text {sub }}, t_{\text {super. }}$ The Alfven radius is determined by the following equations:

$$
\begin{aligned}
R_{\mathrm{A}} & =\left(\frac{\mu^{2}}{2 \dot{M}_{\mathrm{acc}} \sqrt{G M_{\mathrm{NS}}}}\right)^{2 / 7} \\
& \approx 8.2 \times 10^{9} \mu_{30}^{4 / 7} n^{-2 / 7} V_{6}^{6 / 7} \mathrm{~cm}, R_{\mathrm{A}}<R_{\mathrm{G}}, \\
R_{\mathrm{A} 2} & =\left(\frac{4 \mu^{2} G^{2} M_{\mathrm{NS}}^{2}}{\dot{M}_{\mathrm{acc}} V^{5}}\right)^{1 / 6} \\
& \approx 7.6 \times 10^{11} \mu_{30}^{1 / 3} n^{-1 / 6} V_{6}^{-1 / 3} \mathrm{~cm}, R_{\mathrm{A} 2}>R_{\mathrm{G}} .
\end{aligned}
$$

In the later case the equation for the critical period is:

$$
P_{\mathrm{ss} 2}=2 \pi\left(\frac{2 \mu^{2}}{\dot{M}_{\mathrm{acc}} V^{5}}\right)^{1 / 4} \approx 2.5 \times 10^{6} \mu_{30}^{1 / 2} n^{-1 / 4} V_{6}^{-1 / 2} \mathrm{~s} .
$$

For systems with $R_{\mathrm{A}}>R_{\mathrm{G}}$ durations of the propeller substages are different from Eqs. (3) and (4). These systems later appear not as normal accretors but as georotators and so we do not take this effect into account. Study of georotators in binaries is a separate (and probably promising) subject. At that stage there is the possibility of particular "magnetic accretion", see Rutledge (2003).

In the discussed type of binaries there are possibilities for episodes of accretion. As low-mass stars can have episodes of eruptive activity, it is possible that NSs can accrete matter due to fluctuations in the stellar wind. Post main sequence stages are relatively short, so no significant spin-down is expected. There is just a rapid increase of the accretion rate: an increase of $\dot{M}_{*}$ and decrease of $V_{\text {wind }}$ both work to increase $\dot{M}_{\text {acc }}$. Nonaccreting systems, which are situated in the $t_{\mathrm{E}}-t_{\mathrm{P}}$-plane close to the line $t_{\mathrm{E}}=2.3 \times 10^{9} \mathrm{yr}$ (like model 1, 5,9 in Fig. 1) can start to accrete if $\dot{M}_{\text {acc }}$ is increased when the star leaves the main sequence but the system is still detached. Systems with highly eccentric orbits can have episodes of accretion in periastron.

As in the case of isolated NSs, many NSs in pre-LMXBs (at least on the main sequence) are not at the stage of stable wind accretion. Accretion is possible when the donor is at the stage of the main sequence if a NS is strongly magnetized or in close binaries.

Acknowledgements. The author thanks Roberto Turolla and Aldo Treves for discussions and detailed comments on the text and the unknown referee for helpful criticism.

\section{References}

Belczynski, R., \& Taam, R. E. 2003 [arXiv:astro-ph/0311287] Colpi, M., Turolla, R., Zane, S., \& Treves, A. 1998, ApJ, 501, 252

Davies, R. E., \& Pringle, J. E. 1981, MNRAS, 196, 209

Ikhsanov, N. R. 2003, A\&A, 399, 1147

Lipunov, V. M. 1992, Astrophysics of neutron stars (New York Berlin: Springer-Verlag)

Livio, M., Xu, C., \& Frank, J. 1998, ApJ, 492, 298

Perna, R., Narayan, R., Ribicki, G., Stella, L., \& Treves, A. 2003, ApJ, 594, 936

Popov, S. B., \& Prokhorov, M. E. 2000, A\&A, 357, 164

Popov, S. B., Colpi, M., Treves, A., et al. 2000, ApJ, 530, 896

Popov, S. B., Treves, A., \& Turolla, R. 2003, in Proc. 4th AGILE workshop, in press [arXiv: astro-ph/0310416]

Prokhorov, M. E., Popov, S. B., \& Khoperskov, A. V. 2002, A\&A, 381,1000

Rutledge, R. E. 2003, ApJ, 553, 796

Shakura, N. I. 1975, Sov. Astron. Lett., 1, 223

Willems, B., \& Kolb, U. 2003, MNRAS, 343, 949 